\title{
Modeling And PID Control Of A Kind Of Airship Vehicle In Stratosphere Guangbin $\mathrm{Wu}^{1, \mathrm{a}}$, Guoqiang Liang ${ }^{1, \mathrm{~b}}$, Huali $\mathrm{Wu}^{1, \mathrm{c}}$ and Junwei Lei $\mathrm{i}^{1, \mathrm{~d}}$
}

Department of control engineering, Naval aeronautical and astronautical University, Yanti, 264001

demail: leijunwei@126.com

Keywords: airship; pitch channel; adaptive; PID; stability

\begin{abstract}
The airship modeling is the basic of the airship control problem. Based on airship modeling of pitching channel of airship and its nonlinear model, an traditional PID attitude angle controller of airship is designed. Finally, this control method is proved to be effective by numerical simulation.
\end{abstract}

\section{Introduction}

The advantages of airship make airship become more popular in regional high resolution warning, monitoring of territorial, reconfigurable communication, navigational positioning and other fields. Many countries regard airship plan as an important content of the improvement of the efficiency of aero-weapons system, and has studied airship for many years.

In 1998, Brazil AURORA project team of stratospheric airship published initial literature[1]. Literature[2] has given YEZ-2A airship's six degree-of-freedom model that has been confirmed by test flight, and has analysed the part of dynamic response. The course of the flight is divided into five parts including take off, cruise, wheel, suspension and land in literature[3], and corresponding controllers are designed in traditional PID control method.

\section{Model Description}

Based on the previous work, the pitch channel model of airship can be described as follows:

$$
M \dot{x}=f(x)+g(x) u
$$

And $x=\left[\begin{array}{llllll}u & w & q & \theta & x & z\end{array}\right], M_{\text {satisfies }}$

$$
M^{-1}=\left[\begin{array}{llllll}
a_{11} & & a_{13} & & & \\
& a_{22} & & & & \\
a_{31} & & a_{33} & & & \\
& & & 1 & & \\
& & & & 1 & \\
& & & & & 1
\end{array}\right]
$$

The definition of $a_{i j}$ see the definition of $M$ in previous work.[4-5]

Choose the expect value of all states $u, w, q, \theta, x, z$ are $u^{d}, w^{d}, q^{d}, \theta^{d}, x^{d}, z^{d}$,Define the error variable $e=x-x^{d}, \dot{e}=\dot{x}$, then it hold

Use the inverse matrix of $M$

$$
M \dot{e}=f(x)+g(x) u
$$

$$
\dot{e}=M^{-1} f(x)+M^{-1} g(x) u
$$

To make it convenient for reading, some functions can be written as follows 


$$
f(x)=\left[\begin{array}{l}
f_{1} \\
f_{2} \\
f_{3} \\
f_{4} \\
f_{5} \\
f_{6}
\end{array}\right] u=\left[\begin{array}{ll}
u_{1} & u_{2}
\end{array}\right]^{T}
$$

where

$$
\left[\begin{array}{l}
f_{1} \\
f_{2} \\
f_{3} \\
f_{4} \\
f_{5} \\
f_{6}
\end{array}\right]=\left[\begin{array}{c}
-\left(m+m_{33}\right) w q+Q\left[C_{X 1} \cos ^{2} \alpha+C_{X 2} \sin (2 \alpha) \sin (\alpha / 2)\right. \\
\left(m+m_{11}\right) q u+m a_{z} q^{2}+Q\left[C_{z 1} \cos (\alpha / 2) \sin (2 \alpha)+C_{z 2} \sin (2 \alpha)+C_{z 3} \sin (\alpha) \sin (\alpha)\right] \\
-m a_{z} w q(-r v)+Q\left[C_{M 1} \cos (\alpha / 2) \sin (2 \alpha)+C_{M 2} \sin (2 \alpha)+C_{M 3} \sin (\alpha) \sin (\alpha)\right]-a_{z} \sin \theta W \\
q \\
u \cos \theta+w \sin \theta \\
-u \sin \theta+w \cos \theta
\end{array}\right]
$$

Define

$$
M^{-1} f(x)=\left[\begin{array}{c}
f_{a 1} \\
f_{a 2} \\
f_{a 3} \\
f_{a 4} \\
f_{a 5} \\
f_{a 6}
\end{array}\right]=\left[\begin{array}{c}
a_{11} f_{1}+a_{13} f_{3} \\
a_{22} f_{2} \\
a_{31} f_{1}+a_{33} f_{3} \\
f_{4} \\
f_{5} \\
f_{6}
\end{array}\right]
$$

And

$$
g(x) u=\left[\begin{array}{c}
u_{2} \\
k_{1} u_{1} \\
k_{2} u_{1} \\
0 \\
0 \\
0
\end{array}\right]
$$

Then the system can be written as follows[6-8]

$$
\left[\begin{array}{c}
\dot{u} \\
\dot{w} \\
\dot{q} \\
\dot{\theta} \\
\dot{x} \\
\dot{z}
\end{array}\right]=\left[\begin{array}{c}
f_{a 1} \\
f_{a 2} \\
f_{a 3} \\
f_{a 4} \\
f_{a 5} \\
f_{a 6}
\end{array}\right]+\left[\begin{array}{c}
a_{11} u_{2}+a_{13} k_{2} u_{1} \\
a_{22} k_{1} u_{1} \\
a_{31} u_{2}+a_{33} k_{2} u_{1} \\
0 \\
0 \\
0
\end{array}\right]
$$

\section{The PID Control Design For Attitude Control System}

When airship gets disturbed, attitude is the most vulnerable. Attitude control can correct deviation of attitude angle, on the other hand, it can control attitude angle track expected value[9]. 


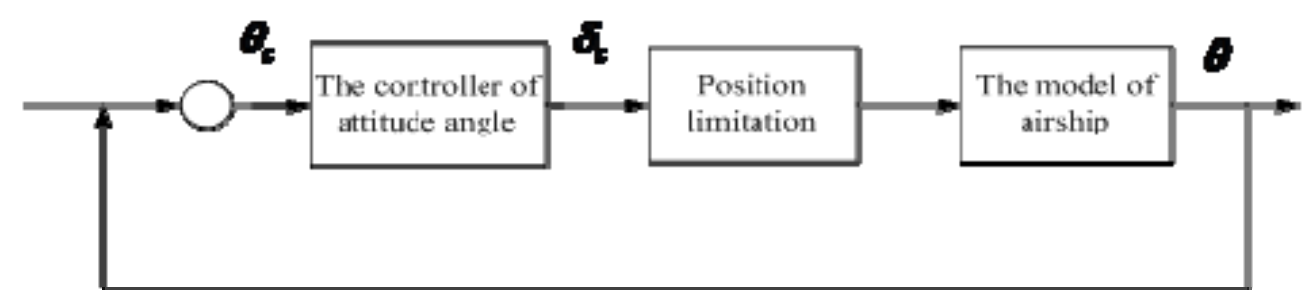

Fig 1 schematic diagram the control loop of attitude angle

Design:

$$
\delta_{e}=K_{p}\left(\theta_{d}-\theta\right)+K_{i} \int_{0}^{t}\left(\theta_{d}-\theta\right) d t+K_{d}\left[\frac{d\left(\theta_{d}-\theta\right)}{d t}\right]
$$

$\theta_{d}$ is expected angle of pitch[10-11]. choose

$$
\begin{aligned}
K_{p} & =0.25 \\
K_{i} & =0.0015 \\
K_{d} & =1
\end{aligned}
$$

\section{Numerical Simulation}

Assume $u=20 \mathrm{~m} / \mathrm{s}, T_{m}=5000 \mathrm{~N}$, initial position $x_{e}=y_{e}=z_{e}=0$. Other quantity of state is 0 , choose $\theta_{d}=10^{\circ}[12-13]$, simulation results are as

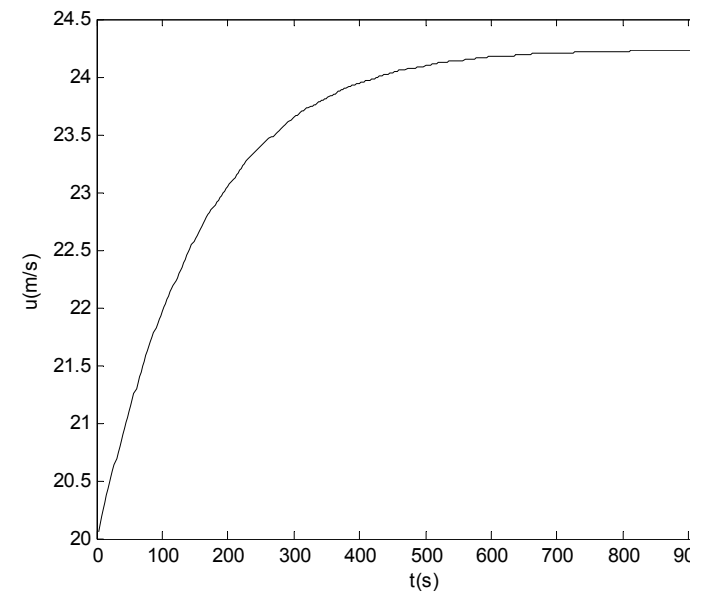

Fig.2 Forward Velocity

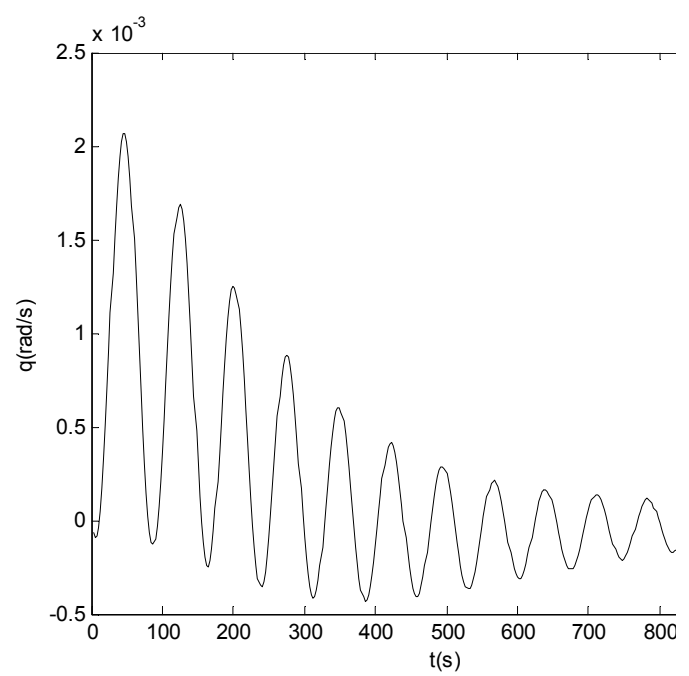

Fig. 4 Angle Velocity

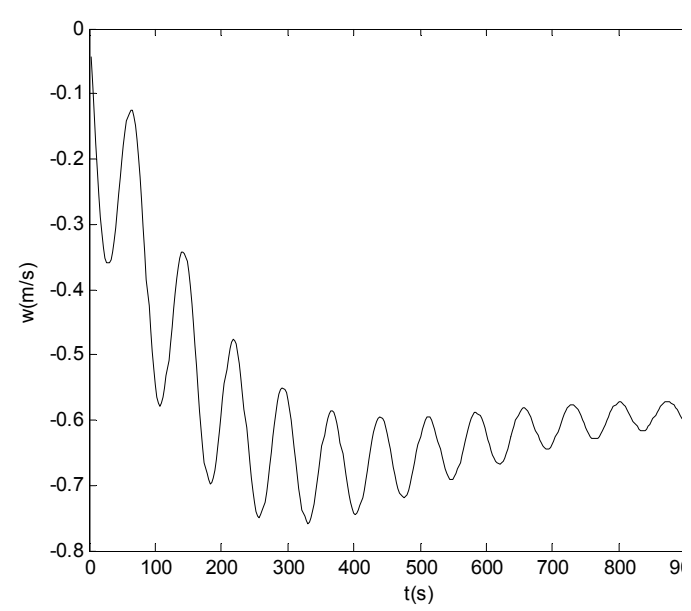

Fig.3 Vertical Velocity

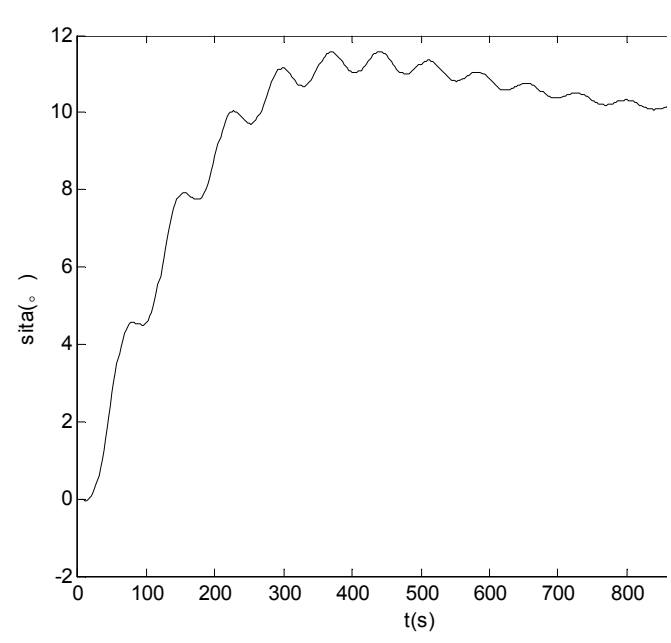

Fig.5 Pitch Angle 


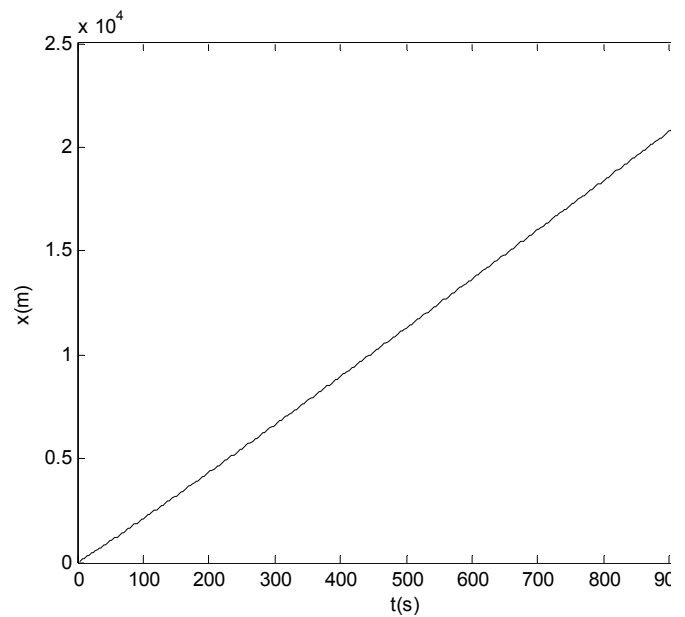

Fig. 6 Flying Distance

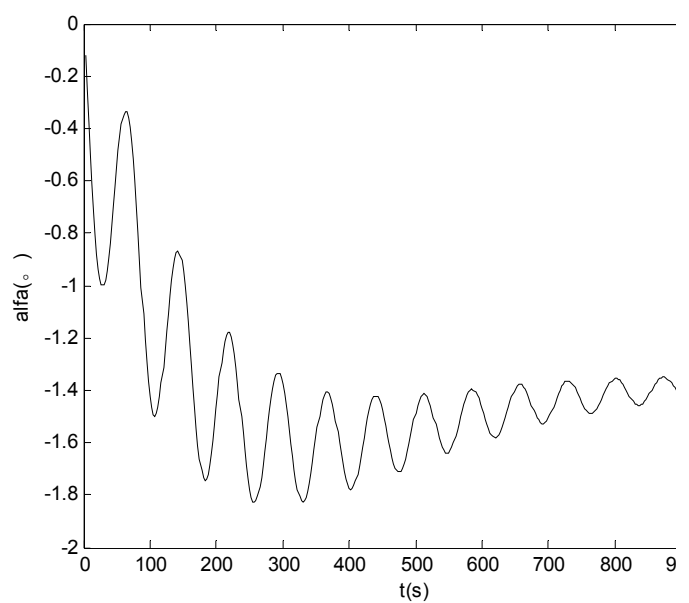

Fig. 8 Pitch Actuator Angle

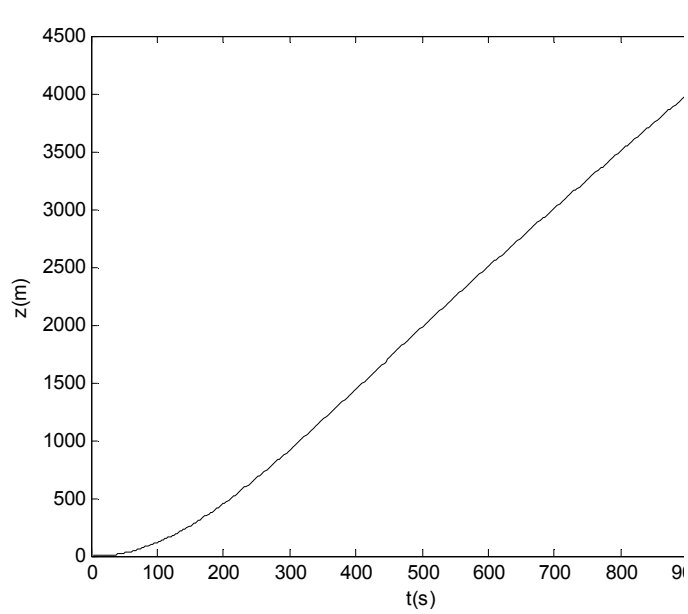

Fig. 7 Height

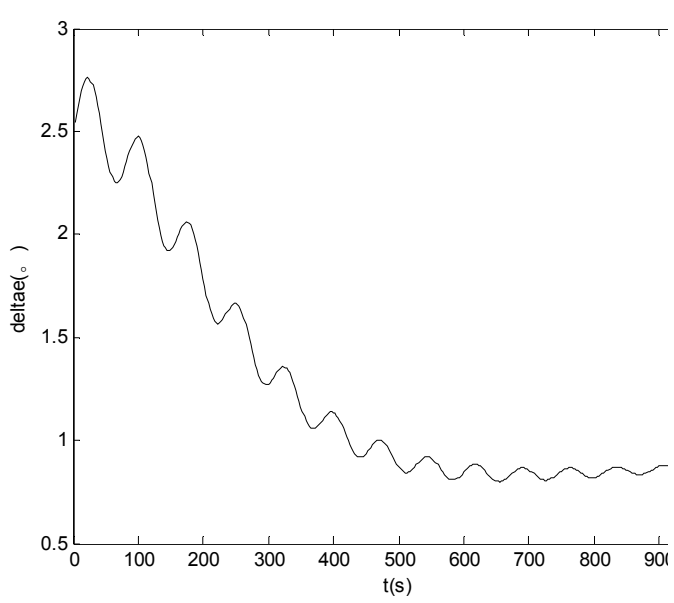

Fig.9 $\delta_{e}$

The simulation results show that airship can follow the tracks of expected angle of pitch in 1000s, and be stable.

\section{Conclusion}

In this paper, a nonlinear model of pitching channel of airship has been established, and this paper has used an traditional PID control scheme to control the angle of pitch of airship. The simulation results show that airship can follow the tracks of expected angle of pitch in 1000 s, and be stable.

\section{References}

[1] Elfes A., S.Bergerman, M.Ramos. A semi-autonomous robotic airship for environmental monitoring missions. IEEE International Conference on Robotics and Automation. 1998. Leuven, Belgium.

[2] Gomes S B V, Romos J J G. Airship Dynamic Modeling for Autonomous Operation. Proceedings of the 1998 IEEE International Conference on Robotics and Automation. 1998, 3462-3467.

[3] de Paiva E C, Bueno S S, Gomes S B V. A Control System Development Environment for AURORA's Semi-Autonomous Robotic Airship. IEEE Internatioal Conference on Robotics \& Automation. Detroit, MI, USA, 1999, 2328-2335. 
[4] Azinheira, J.R., A.Moutinho, E.C. De Paiva, Airship hover stabilization using a backstepping control approach. 2006.29(4):903-914.

[5] Benjovengo F P, Paiva E C. Nonlinear control approaches for an autonomous unmanned robotic airship[C]. In: 17th AIAA Aviation Technology Conference, Belfast, Northern Ireland, 2007

[6] Lee S. Lee H. Backstepping apporoach of trajectory tracking control for the mid-altitude unmanned airship. AIAA 2007-6319,2007.

[7] Repoulias F, Papadopoulos E. Robotic airship trajectory tracking control using a backstepping methodology.[C] In:2008 IEEE International Conference on Robotics and Automation, Pasadena, CA, USA, 2008

[8] Azinheira, J.R., A.Moutinho, Hover Control of an UAV With Backstepping Design Including Input Saturations[J]. IEEE Transactions on Control System Technology, 2008.16(3):517-526

[9] Azinheira J R, Moutinho A, Paiva E C. A backstepping controller for path-tracking of an underactuated autonomous aitship.[J] International Journal of Robust and Nonlinear Control, 2009, 19(4):418-441

[10] Benjovengo F P, Paiva E C. Sliding mode control approaches for an autonomous unmanned airship. AIAA 2009-2869, 2009

[11] Park, C.S., H.Lee, M.J.Tahk, et al. Airship control using neural network augmented model inversion[J]. IEEE Conference on Control Applications. 2003

[12] Xie, S.R., J.Luo, J.J.Rao, et al. Computer Vision-based Navigation and Predefined Track Following Control of a Small Robotic Ariship. 2007.33(3):286-291.

[13] Rao, J., Z.Gong, J.Luo, et al., Robotics airship mission path-following control based on ANN and human operator's skill. 2007.29:5-15. 